\title{
Construction Quality Residential Project Based on Artificial Neural Network
}

\author{
Yue Yue1, Jiaye $\mathrm{Wu}^{2 *}$, Shuyang Liu1, Yingqian Zhang ${ }^{3}$ \\ ${ }^{1}$ School of Management of Sichuan University of Science \& Engineering, Zigong, China \\ ${ }^{2}$ Sichuan Shengtuo Testing Technology Co., LTD., Zigong, China \\ ${ }^{3}$ School of Civil Engineering of Sichuan University of Science \& Engineering, Zigong, China \\ Email:*616181649@qq.com
}

How to cite this paper: Yue, Y., $\mathrm{Wu}$ J.Y., Liu, S.Y. and Zhang, Y.Q. (2019) Construction Quality Residential Project Based on Artificial Neural Network. Open Access Library Journal, 6: e5681. https://doi.org/10.4236/oalib.1105681

Received: August 8, 2019

Accepted: August 26, 2019

Published: August 29, 2019

Copyright (c) 2019 by author(s) and Open Access Library Inc.

This work is licensed under the Creative

Commons Attribution International

License (CC BY 4.0).

http://creativecommons.org/licenses/by/4.0/

\section{Open Access}

\begin{abstract}
Engineering quality evaluation is an important part of engineering quality management. However, China has not yet formed a systematic engineering quality evaluation system. Aiming at such problems, a comprehensive evaluation method based on artificial neural network for residential project construction quality is proposed. Based on the analysis of the quality influencing factors in the construction process of residential projects, a construction quality grade index system was established. Based on the index system, the Analytic Hierarchy Process (AHP) was used to qualitatively and quantitatively analyze various factors, combined with expert scoring. The method obtains quantitative decision-making results; the multi-index comprehensive evaluation method using artificial neural network and AHP method simulates the expert to evaluate the construction quality of residential buildings and compares the model evaluation results of construction quality with the evaluation results of experts, and analyzes artificial nerves. The case analysis of a residential project was carried out to verify the effectiveness and feasibility of the method, and the superiority of the artificial neural network in comprehensive evaluation is demonstrated.
\end{abstract}

\section{Subject Areas}

Engineering Management

\section{Keywords}

Artificial Neural Network, Quality Evaluation, Analytic Hierarchy Process

\section{Introduction}

In recent years, China's construction industry has experienced rapid develop- 
ment, and the overall level of residential project quality has been greatly improved. The quality of residential projects was related to the sustained and healthy development of the country's social economy. It is particularly important to strengthen the quality management of residential projects and overcome the common problems of residential quality. Engineering quality evaluation is an important part of project quality management. How to use scientific and reasonable methods to control and evaluate the quality of residential construction, to improve the overall quality level of residential projects, has become a major issue of common concern at the present stage.

Pim Polesie conducts an in-depth analysis of the status quo of engineering quality and the quality of engineering construction through the evaluation results of the evaluation system, and proposes reasonable quality improvement measures [1]. At present, China has not yet formed a systematic and comprehensive construction project quality evaluation system and model. This leads to the lack of relevant data for the personnel engaged in the quality management of construction engineering to support the evaluation and analysis of engineering quality, so that the specific measures formulated by the quality management personnel are out of touch with the actual situation, resulting in waste of resources and even hidden quality hazards [2]. Zhao Lingyan and Zhao Qun systematically analyzed the insufficiency of AHP in construction engineering, explained the process of establishing model and calculation in AHP, expounded the concept of index weight, and determined the method of determining weight coefficient by using AHP. From the consistency test results, a satisfactory result was obtained [3].

Based on the above research, this paper conducts further research on the comprehensive evaluation method of residential construction quality, optimizes the factors of residential construction quality, establishes the construction quality index system of residential construction and combines AHP method and expert scoring method. After obtaining the quantitative decision results, the multi-index comprehensive evaluation method based on the artificial neural network and AHP method is used to comprehensively judge the construction quality of the residential project, and the network prediction results are obtained and the quality of the construction is judged. Finally, model evaluation results of residential construction quality and the evaluation results of experts are compared and analyzed, and an example analysis of a residential project is carried out.

\section{Establishment of the Quality Index System for Residential Construction}

At present, the method for judging the quality of residential construction is mainly judged by the workers and experts according to the requirements and test criteria of the residential construction documents, the degree of automation is low and the efficiency is low; and the workers and experts are vulnerable to the feasibility determination. People's disturbance of subjective consciousness, poor 
reliability and accuracy. The key to solving the problem of quality control and evaluation of residential construction is to establish a feasible, comprehensive and scientific evaluation method.

The factors influencing the construction quality of residential projects are complex and diverse, and a large number of them are proposed. Based on the comprehensive analysis and analysis, the evaluation index system of residential engineering construction quality grade is proposed, as shown in Figure 1.

The evaluation index system for the quality of residential construction shown in Figure 1 is a hierarchical analysis system established after considering the construction process and results of the residential project from many aspects, multiple angles and multiple levels. The establishment process fully follows the concise and relevant, Accurate assembly and hierarchical principles. The system is divided into three levels: the target layer, the criterion layer, and the indicator layer; the highest level is also the target layer to represent the ultimate goal of solving the problem: to judge the quality of the residential construction; the middle layer is also the criterion layer. It represents the intermediate link through which the predetermined goal is achieved. The bottom layer, the indicator layer, represents the specific solution to the problem. It is the key factor for the detailed description of the middle layer after analyzing the middle layer factors. At the same time, the criterion layer and the index layer also constitute a two-level evaluation comprehensive system of the residential engineering construction quality level evaluation index system, that is, the first-level evaluation

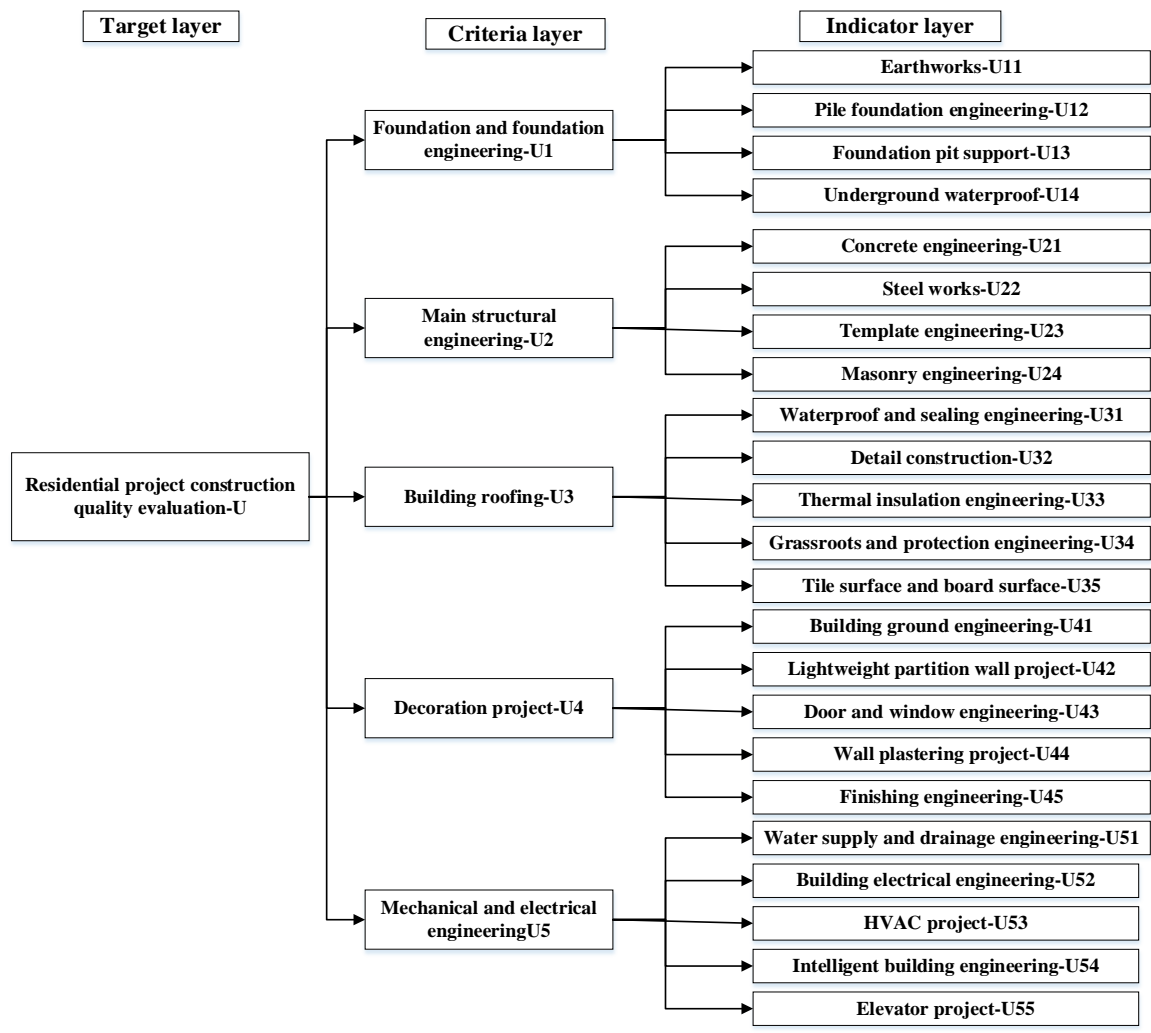

Figure 1. Residential project construction quality evaluation (figure caption). 
factor set and the second-level evaluation factor set. After naming and quantifying the index numbers in the two-level evaluation factor level, the evaluation value of each index is the data source of the evaluation index system of the entire residential construction quality level.

Before quantifying the influencing factors of the quality assessment of residential construction, it is first necessary to establish a grade evaluation criterion for the comprehensive quantitative results of various indicators. After comprehensively considering the indicators in the secondary evaluation factor set that affects the construction quality of the residential project, the quality evaluation criteria for the construction of the residential building as shown in Table 1 are established. The analytic hierarchy process and the expert scoring method determine the scores of the grades of the grades of the quality grades of residential construction. The five quality grade standards for residential construction quality evaluation are \{excellent, good, medium, qualified, unqualified\}, and the representative score interval is the comprehensive score interval after quantification of each index factor in the residential construction quality assessment. Comparing the comprehensive result value of the residential construction quality of the project to be evaluated with the quality evaluation standard of Table 1, the level of the quality of the residential project to be evaluated can be further determined.

\section{AHP Decision Result}

The difficulty in evaluating the evaluation index system of residential construction quality grade is: 1) the multi-objective evaluation object, the comprehensive evaluation is difficult; 2) the qualitative and quantitative indicators coexist, for the subjective with the user or the appraiser only Feeling qualitative indicators related to experience, due to the lack of a clear quantitative representation, it is difficult to judge; 3 ) the more indicators, the more complicated the problem.

Analytic Hierarchy Process (AHP) is a multi-criteria decision-making method combining qualitative and quantitative analysis. After using (AHP) method to decompose related elements of decision-making problems of residential construction quality into target layer, criterion layer and index layer, The subjective judgment of human beings is objectively quantified with a certain scale; on this basis, qualitative analysis and quantitative analysis are carried out, and finally

Table 1. Construction quality rating standard.

\begin{tabular}{ccc}
\hline Category & Cronbach's Alpha is based on standardized items & Number of items \\
\hline I & Excellent & {$[0.9-0.8]$} \\
II & Good & {$[0.8-0.7]$} \\
III & Medium & {$[0.7-0.6]$} \\
IV & Qualified & {$[0.6-0.8]$} \\
V & Unqualified & {$[0.5-0.0]$} \\
\hline
\end{tabular}


the problem is reduced to the problem of determining the relative importance weight of the lowest level relative to the highest level [4]. The steps of analyzing the various factors of cable laying quality evaluation by AHP method, and obtaining the decision results of various factors and indicators are as follows:

Step one constructs a judgment matrix.

The elements of each layer in the evaluation index system of the construction quality of the residential project are compared with each other, and the judgment matrix of the two elements can be obtained. The $1-9$ scale method is used to quantify the decision judgment, and the experts analyze and determine two kinds of numerical judgment matrices: 1) the judgment matrix of the criterion layer for the target layer $U$;2) the judgment matrix of the index layer for the criterion layer $U-U_{i}$, among them $i=\{1,2,3,4,5\}$.

Step two Consistency test.

In order to prevent the determination of the relative importance of each indicator, there will be uncoordinated or even contradictory results, and a consistency check is required. According to the relationship between the matrix and the corresponding eigenvalues in the matrix theory, the characteristics of the eigenvalue can largely reflect the characteristics of the matrix to which it belongs. Therefore, the indicator that the measurement matrix deviates from the consistency is represented by the eigenvalue ( $n=$ matrix order)

$$
C I=\frac{\lambda_{\max }-n}{n-1}
$$

The larger the value of $C I$, the worse the consistency test result of the matrix is, and the more uncoordinated between the indicators; on the contrary, the better the consistency test and the coordination of the indicators.

$$
C R=\frac{C I}{R I}
$$

As shown in Equation (2), in order to characterize the matrix random consistency ratio, the ratio of $C I$ to $R I$ (mean random consistency index) is selected to determine and denoted as $C R$. Wherein, the value of $R I$ is obtained from Table 2 according to the order of the matrix.

With 0.1 as the limit, the meaning of the $C R$ value can be divided into two cases:

$$
\left\{\begin{array}{l}
(\text { I }) C R<0.1 \text {, The matrix has satisfactory consistency } \\
(\text { II }) C R \geq 0.1 \text {, Make adjustments until satisfaction }
\end{array}\right.
$$

\section{BP Artificial Neural Network Evaluation Method}

Neural Network (NN) is the abbreviation of Artificial Neural Network (ANN), which is a mathematical model or computational model simulating the structure and function of biological neural network [5].

The most commonly used algorithm of Neural Network is Back Propagation (BP), which consists of an input layer, a hidden layer and an output layer (Figure 2). Its main idea is: 
Table 2. $R I$ (mean random consistency indicator) value table.

\begin{tabular}{cccccccc}
\hline Order & 1 & 2 & 3 & 4 & 5 & 6 & 7 \\
\hline R value & 0 & 0 & 0.58 & 0.88 & 1.12 & 0.24 & 0.32 \\
\hline
\end{tabular}

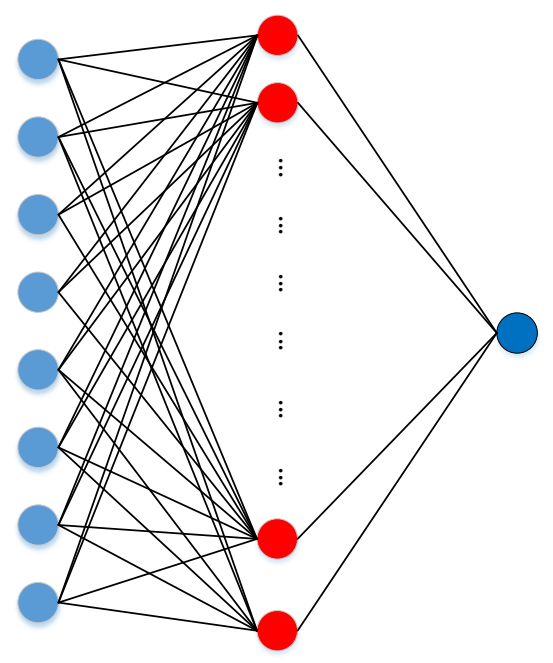

\section{Input Layer Hidden Layer Output Layer}

Figure 2. Multi-layer neural network.

1) Since the output result of NN has an error with the actual result, the error between the estimated value and the actual value is calculated, and the error is propagated back from the output layer to the hidden layer until it is propagated to the input layer;

2) In the process of $\mathrm{BP}$, the value of various parameters is adjusted according to errors;

3) Continuously iterate the above process until convergence occurs.

Multi-layer neural networks can also be implemented by least square method

Learning:

$$
E(\omega)=\sum_{i=1}^{N}\left(y_{i}-o_{i}\right)^{2}
$$

where,

$$
o_{i}=\frac{1}{1+\mathrm{e}^{(-\omega \cdot x)_{i}}}
$$

Gradient Descent Method is adopted, there is

$$
\begin{aligned}
& \frac{\partial E}{\partial \omega_{j}}=2 \sum\left(y_{i}-o_{i}\right) \frac{\partial\left(y_{i}-o_{i}\right)}{\partial \omega_{j}} \\
& \frac{\partial E}{\partial \omega_{j}}=2 \sum\left(y_{i}-o_{i}\right) \frac{\partial\left(y_{i}-o_{i}\right)}{\partial \omega_{j}}
\end{aligned}
$$

where, 


$$
\begin{gathered}
\frac{\partial y_{i}}{\partial \omega_{j}}=0 \\
\frac{\partial o_{i}}{\partial \omega_{j}}=\frac{\partial o_{i}}{\partial h_{i}} \frac{\partial h_{i}}{\partial \omega_{j}}=o_{i}\left(1-o_{i}\right) a_{j} \\
\frac{\partial o_{i}}{\partial \omega_{j}}=\frac{\partial o_{i}}{\partial h_{i}} \frac{\partial h_{i}}{\partial \omega_{j}}=o_{i}\left(1-o_{i}\right) a_{j}
\end{gathered}
$$

where, $a_{j}$ is the output of Unit $J$ in previous layer.

Multiply the obtained gradient by the penalty term $\eta$, and correct the weight sequence to prevent over-fitting of the model, i.e.:

$$
\begin{aligned}
& \omega_{j}-\eta \frac{\partial E}{\partial \omega_{j}} \rightarrow \omega_{j} \\
& \omega_{j}-\eta \frac{\partial E}{\partial \omega_{j}} \rightarrow \omega_{j}
\end{aligned}
$$

Repeat iterating the above process until the error meets the requirement.

\section{Case Analysis}

The above method is applied to test the construction quality of a residential project and verify the feasibility (Tables 3-8).

Each expert multiplies the weight value of each sub-item, and finally the comprehensive value of the evaluation of the quality of the residential construction by 20 experts is obtained. The results are shown in Table 9 .

Fi Finally, samples with numbers $1-15$ are used as training sets and $15-20$ are used as test sets. As shown in Table 10. The training set data (sample number 1 - 15) shows that the relative error of the output result of the network is very small, and the output result is almost consistent with the expected result, which proves that the network has reached the accuracy requirement training; the serial number is $16-20$. The output of the simulation evaluation of the five sets of test set data and the expected output are shown in Table 10.

As can b As can be seen from Table 10, the relative error between the output value and the expected value obtained by the neural network is controlled at a fraction of a few percents to a few percent, which is almost identical to the evaluation result of the desired output. Figure e shows a comparison of the results.

Table 3. Judgment matrix $U$.

\begin{tabular}{ccccccc}
\hline & $U_{1}$ & $U_{2}$ & $U_{3}$ & $U_{4}$ & $U_{5}$ & Weights \\
\hline$U_{1}$ & 1 & $1 / 2$ & 2 & 3 & 3 & 0.251 \\
$U_{2}$ & 2 & 1 & 3 & 5 & 4 & 0.422 \\
$U_{3}$ & $1 / 2$ & $1 / 3$ & 1 & 3 & 2 & 0.162 \\
$U_{4}$ & $1 / 3$ & $1 / 5$ & $1 / 3$ & 1 & $1 / 2$ & 0.066 \\
$U_{5}$ & $1 / 3$ & $1 / 4$ & $1 / 2$ & 2 & 1 & 0.099 \\
\hline
\end{tabular}

$\lambda_{\max }=5.0859, C I=0.0214, \mathrm{RI}=1.12, C R=0.0191$. 
Table 4. Judgment matrix $U_{1}$.

\begin{tabular}{cccccc}
\hline & $U_{11}$ & $U_{12}$ & $U_{13}$ & $U_{14}$ & Weights \\
\hline$U_{11}$ & 1 & $1 / 4$ & $1 / 3$ & $1 / 2$ & 0.095 \\
$U_{12}$ & 4 & 1 & 2 & 3 & 0.467 \\
$U_{13}$ & 3 & $1 / 2$ & 1 & 2 & 0.278 \\
$U_{14}$ & 2 & $1 / 3$ & $1 / 2$ & 1 & 0.160 \\
\hline
\end{tabular}

$\lambda_{\max }=4.0310, C I=0.0103, R I=0.88, C R=0.0117$.

Table 5. Judgment matrix $U_{2}$.

\begin{tabular}{cccccc}
\hline & $U_{21}$ & $U_{22}$ & $U_{23}$ & $U_{24}$ & Weights \\
\hline$U_{21}$ & 1 & $1 / 3$ & 2 & 3 & 0.233 \\
$U_{22}$ & 3 & 1 & 4 & 5 & 0.545 \\
$U_{23}$ & $1 / 2$ & $1 / 4$ & 1 & 2 & 0.138 \\
$U_{24}$ & $1 / 3$ & $1 / 5$ & $1 / 2$ & 1 & 0.084 \\
\hline
\end{tabular}

$\lambda_{\max }=4.0511, C I=0.0170, R I=0.88, C R=0.0193$.

Table 6. Judgment matrix $U_{3}$.

\begin{tabular}{ccccccc}
\hline & $U_{31}$ & $U_{32}$ & $U_{33}$ & $U_{34}$ & $U_{35}$ & Weights \\
\hline$U_{31}$ & 1 & 3 & 2 & 3 & 4 & 0.396 \\
$U_{32}$ & $1 / 3$ & 1 & $1 / 2$ & 1 & 2 & 0.135 \\
$U_{33}$ & $1 / 2$ & 2 & 1 & 3 & 4 & 0.277 \\
$U_{34}$ & $1 / 3$ & 1 & $1 / 3$ & 1 & 1 & 0.108 \\
$U_{35}$ & $1 / 4$ & $1 / 2$ & $1 / 4$ & 1 & 1 & 0.084 \\
\hline
\end{tabular}

$\lambda_{\max }=5.0811, C I=0.0203, R I=1.12, C R=0.0181$.

Table 7. Judgment matrix $U_{4}$.

\begin{tabular}{ccccccc}
\hline & $U_{41}$ & $U_{42}$ & $U_{43}$ & $U_{44}$ & $U_{45}$ & Weights \\
\hline$U_{41}$ & 1 & $1 / 3$ & $1 / 2$ & 1 & 2 & 0.130 \\
$U_{42}$ & 3 & 1 & 2 & 4 & 5 & 0.422 \\
$U_{43}$ & 2 & $1 / 2$ & 1 & 3 & 4 & 0.267 \\
$U_{44}$ & 1 & $1 / 4$ & $1 / 3$ & 1 & 2 & 0.113 \\
$U_{45}$ & $1 / 2$ & $1 / 5$ & $1 / 4$ & $1 / 2$ & 1 & 0.067 \\
\hline
\end{tabular}

$\lambda_{\max }=5.1833, C I=0.0458, R I=1.12, C R=0.0409$.

The distance between the two fold lines in Figure e is very small, indicating that the BP neural network prediction results are very similar to the expected results (Figure 3 ). 
Table 8. Judgment matrix $U_{5}$.

\begin{tabular}{ccccccc}
\hline & $U_{51}$ & $U_{52}$ & $U_{53}$ & $U_{54}$ & $U_{55}$ & Weights \\
\hline$U_{51}$ & 1 & $1 / 2$ & 2 & 4 & 3 & 0.274 \\
$U_{52}$ & 2 & 1 & 3 & 4 & 3 & 0.392 \\
$U_{53}$ & $1 / 2$ & $1 / 3$ & 1 & 2 & 2 & 0.154 \\
$U_{54}$ & $1 / 4$ & $1 / 4$ & $1 / 2$ & 1 & $1 / 2$ & 0.073 \\
$U_{55}$ & $1 / 3$ & $1 / 3$ & $1 / 2$ & 2 & 1 & 0.107 \\
\hline
\end{tabular}

$\lambda_{\max }=5.1044, C I=0.0261, R I=1.12, C R=0.0233$.

Table 9. Comprehensive value of construction project quality evaluation.

\begin{tabular}{ccccccccccc}
\hline Number & 1 & 2 & 3 & 4 & 5 & 6 & 7 & 8 & 9 & 10 \\
\hline Value & 0.859 & 0.870 & 0.847 & 0.855 & 0.847 & 0.874 & 0.826 & 0.873 & 0.723 & 0.667 \\
Number & 11 & 12 & 13 & 14 & 15 & 16 & 17 & 18 & 19 & 20 \\
Value & 0.801 & 0.891 & 0.769 & 0.895 & 0.887 & 0.869 & 0.899 & 0.530 & 0.895 & 0.864 \\
\hline
\end{tabular}

Table 10. Simulation results and relative error.

\begin{tabular}{ccccccccccc}
\hline Number & 1 & 2 & 3 & 4 & 5 & 6 & 7 & 8 & 9 & 10 \\
\hline Training & 0.871 & 0.853 & 0.849 & 0.863 & 0.855 & 0.859 & 0.877 & 0.883 & 0.768 & 0.686 \\
Actual & 0.859 & 0.870 & 0.847 & 0.855 & 0.847 & 0.874 & 0.826 & 0.873 & 0.723 & 0.667 \\
Error & 0.013 & 0.018 & 0.002 & 0.009 & 0.009 & 0.017 & 0.062 & 0.011 & 0.062 & 0.028 \\
Number & 11 & 12 & 13 & 14 & 15 & 16 & 17 & 18 & 19 & 20 \\
Training & 0.747 & 0.884 & 0.619 & 0.883 & 0.872 & 0.867 & 0.890 & 0.442 & 0.882 & 0.884 \\
Actual & 0.801 & 0.891 & 0.769 & 0.895 & 0.887 & 0.869 & 0.899 & 0.530 & 0.895 & 0.864 \\
Error & 0.066 & 0.008 & 0.195 & 0.013 & 0.017 & 0.002 & 0.011 & 0.066 & 0.015 & 0.023 \\
\hline
\end{tabular}

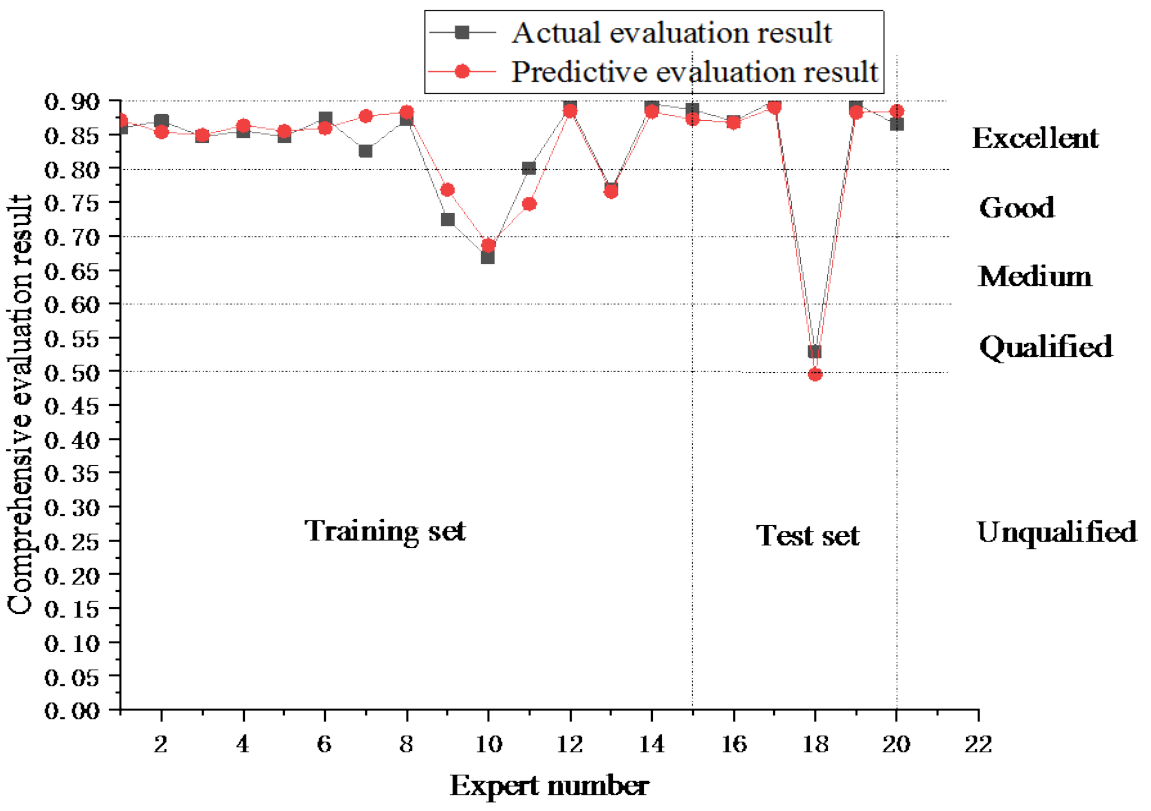

Figure 3. Comparison of predicted results with expected results. 


\section{Conclusion}

Aiming at the evaluation of construction quality of residential projects, this paper establishes a more comprehensive construction quality index system for residential projects, and uses artificial neural network algorithms to learn the knowledge and "experience" of experts, and the simulation experts evaluate the construction quality of residential projects. The artificial neural network automatically calculates and outputs the evaluation results according to the index data of the input network, and does not need to manually determine the weight of each part of the data, which reduces the possibility of being affected by non-objective factors in the evaluation process, and improve the reliability, objectivity and accuracy of the evaluation results. For some obvious errors in the sample, the artificial neural network can be automatically eliminated, and the fault tolerance of the network ensures the accuracy of the whole system, so that the overall fitting degree is very high and the evaluation effect is excellent [6]. The artificial neural network has shown excellent superiority and very broad application prospects in the application of construction project quality assessment. Compared with other evaluation methods, this paper uses artificial neural network to evaluate the construction quality of residential projects with higher efficiency and accuracy, real-time evaluation and control of the construction quality of residential projects, and provides workers for residential project construction. When learning and analyzing databases with large data volumes, the network has obvious advantages over other evaluation methods in terms of accuracy, efficiency and relative error.

\section{Conflicts of Interest}

The authors declare no conflicts of interest regarding the publication of this paper.

\section{References}

[1] Pun, P. (2013) The View of Freedom and Standardization among Managers in Swedish Construction Contractor Projects. International Journal of Project Management, 42, 299-306. https://doi.org/10.1016/j.ijproman.2012.09.010

[2] Gao, H. and Li, H.M. (2002) Application of Fuzzy Comprehensive Evaluation Method in Engineering Quality Risk Analysis. Journal of Xi an University of Science and Technology, 22, 21-23.

[3] Wu, Z.J. and Wang, W.W. (2010) Application of BP Neural Network in Sales Forecast of Stocking Enterprises. Industrial Engineering, 1, 105-107.

[4] Zhai, X. (2015) Slope Deformation Monitoring Method Based on 3D Laser Scanning Technology. China University of Geosciences, Beijing.

[5] Fan, H., Wang, F. and Wang, G.W. (2017) Research Progress in the Laying Method of Hot Water Hot Pipe Gallery. Journal of Huaqiao University (Natural Science), 38, 747-752.

[6] Wang, Y.Z. and Fu, L. (2013) Research on the Countermeasures of Urban Comprehensive Pipeline Charging Based on Game Analysis. Chinese Journal of Underground Space and Engineering, 9, 197-203. 